\title{
Orientation dynamics of dilute functionalized graphene suspensions in oscillatory flow
}

\author{
Giovanniantonio Natale* \\ Department of Chemical and Petroleum Engineering, University of Calgary, 2500 University Drive NW, \\ Calgary, Alberta, Canada T2N $1 N 4$ \\ Naveen K. Reddy \\ Faculty of Industrial Engineering, Hasselt University, Martelarenlaan 42, 3500 Hasselt, Belgium \\ and IMO, IMOMEC, Hasselt University, Wetenschapspark 1, 3590 Diepenbeek, Belgium \\ Robert K. Prud'homme \\ Department of Chemical and Biological Engineering, Princeton University, \\ Princeton, New Jersey 08544, USA \\ Jan Vermant \\ Department of Materials, ETH Zürich, Vladimir-Prelog-Weg 5, Zürich 8093, Switzerland
}

(Received 12 March 2018; published 20 June 2018)

\begin{abstract}
The dynamics of molecularly thin graphene sheets in transient flows are important to understand their behavior in suspension and during processing. Scattering dichroism is used to evaluate changes in the orientation distribution function in dilute suspensions. To evaluate if the graphene sheets behave as flexible sheets or as more rigid flat particles, the results are compared with numerical computations of the single-particle Smoluchowski equation for flat spheroidal particles. In particular, the evolution of the orientation angle in oscillatory flows as a function of increasing amplitude is studied. The results show that even when taking an average rotational diffusivity, the results for the polydisperse graphene sheets show all the hallmarks of the motion of rigid disks, including a frequency doubling of the time-dependent orientation signal. The results indicate that the motion of functionalized graphene sheets in suspension is consistent with flat rigid objects.
\end{abstract}

DOI: 10.1103/PhysRevFluids.3.063303

\section{INTRODUCTION}

The most common rheological measurements to characterize the viscoelastic properties of materials use an oscillatory shear flow that creates a model time-dependent flow. In oscillatory shear flow, a sinusoidal strain $\gamma(t)=\gamma_{0} \sin (\omega t)$ is imposed on the material where $\gamma_{0}$ is the strain amplitude and $\omega$ the angular frequency. The amplitude of the associated shear rate is given by $\dot{\gamma}_{0}=\gamma_{0} \omega$. Here, in addition to the flow timescale (which is equal to $1 / \dot{\gamma}_{0}$ ), a second timescale is set by the imposed frequency called oscillation period $2 \pi / \omega$. For the case of a monodisperse Brownian suspension, two dimensionless groups can be formed by combining the above two timescales $\left(1 / \dot{\gamma}_{0}\right.$ and $2 \pi / \omega)$ together with $\tau$, the timescale associated with the rotational Brownian motion. The first is the Weissenberg number, which is the ratio between the relaxation time and the flow time, $\mathrm{Wi}=\tau \dot{\gamma}_{0}$. The second is the Deborah number, which is the ratio of the relaxation time to the oscillation period,

\footnotetext{
* gnatale@ucalgary.ca
} 
$\mathrm{De}=\tau \omega$. If $\mathrm{Wi}$ and $\mathrm{Wi} / \mathrm{De}$ are larger than 1 , the system is driven out of equilibrium and the simple linear proportionality of the shear stress with the amplitude is lost [1-3]. This regime is called large-amplitude oscillatory shear (LAOS). Recently, LAOS has attracted major interest as a characterization methodology to fully explore material properties as it provides rich information encoded in the shear and normal stress responses. However, it is still challenging to fully understand how microstructural states are linked to the oscillatory response.

A dilute suspension of molecularly thin two-dimensional (2D) particles is used here as a model system to study the relationship between microstructural states and the mechanical response of the system under an oscillatory perturbation. Specifically, the experimental system consists of a polydisperse graphene nanoparticles dispersed in a Newtonian matrix. Polydispersity implies a continuous spectrum of relaxation times in the system. This feature is commonly encountered in nanoparticles suspensions, colloids, bacteria suspensions, and rigid polymers. Hence the relationships between microstructure and overall mechanical response will be shared among these systems. Graphene is a carbon-based material with a unique 2D structure [4]. Scaling arguments from polymer theory for $2 \mathrm{D}$ polymers suggest that self-avoiding surfaces make the flat state dominant for such thin 2D particles, even for small values of bending rigidity [5]. Hence the dynamics of these thin sheets can be expected to be similar to thicker, rigid nonspherical particles. Grapheneous materials are also interesting from a more technological perspective. They possess exceptional transport and mechanical properties [6-9] that are promising for multiple applications: conducting probes or electrodes [10,11], emulsion stabilizer [12], drug sensors [13], and nanocomposites [9,14]. The potential of this material is yet to be fully exploited. The current bottleneck for using graphene suspensions in wet processing is to design and optimize manufacturing processes to obtained controlled orientation and dispersion states at the nanoscale.

In the present work we probe experimentally using linear flow dichroism $[15,16]$ the microstructure evolution in transient oscillatory flows going from small to large strain amplitudes. We compare the orientation dynamics of the thin graphene sheets to a mathematical model for spheroidal nanoparticles. Linear flow dichroism using polarimetric modulation is a rheo-optical characterization technique that is able to measure, with great temporal resolution and exquisite sensitivity, changes in the complex part of the refractive index tensor. These changes for anisotropic particles are directly related to change in the microstructural state [17-22]. Successful applications of this optical technique to determine flow-induced alignment of rodlike and disklike materials have been reported [15,21,23-29] but they have been mainly focusing on steady-state shear flow or simple transient shear flow. In the present work we probe the orientation evolution in transient oscillatory flow for molecularly thin functionalized graphene sheet suspension.

Large-amplitude oscillatory shear has been used to investigate how good classic constitutive rheological relations deal with the onset of nonlinear responses, including power-law fluids [30], the Giesekus model [31-39], the corotational Maxwell and corotational Jeffrey models [40], and the Johnson-Segalman model [31]. To strengthen the link between microstructures and LAOS, micromechanical models have also been numerically computed for microstructural models of entangled polymers [41], dilute and stabilized spherical drops [42], deformable spheres [43], dilute suspensions of dumbbells [44], Brownian rods [45], and active Brownian rods [46]. Analytical solutions obtained via singular perturbation methods have also been reported for the Giesekus model [1], slightly deformed spheroids [2], and dilute suspensions of slender rods [47]. In the present work, we use LAOS as a prototypal transient flow and use the rheo-optical method to measure the orientation evolution of thin graphene disks. To see if these molecularly thin 2D sheets behave as rigid flakes, the results are compared to exact results for rigid thin spheroidal objects, analyzed by modeling the orientation distribution function as a convected quantity [48]. The Fokker-Planck or single-particle Smoluchowski equation is used to describe the orientation state of the systems and it is numerically computed. The comparison between the experimental results on the graphene flakes and the model for spheroidal particles serves to investigate to what extent the molecularly thin graphene sheets behave as rigid flat disks and whether they crumple and fold during flow. In particular, measuring the time-dependent orientation angle will prove to be insightful. For rigid, flat objects, the particle 


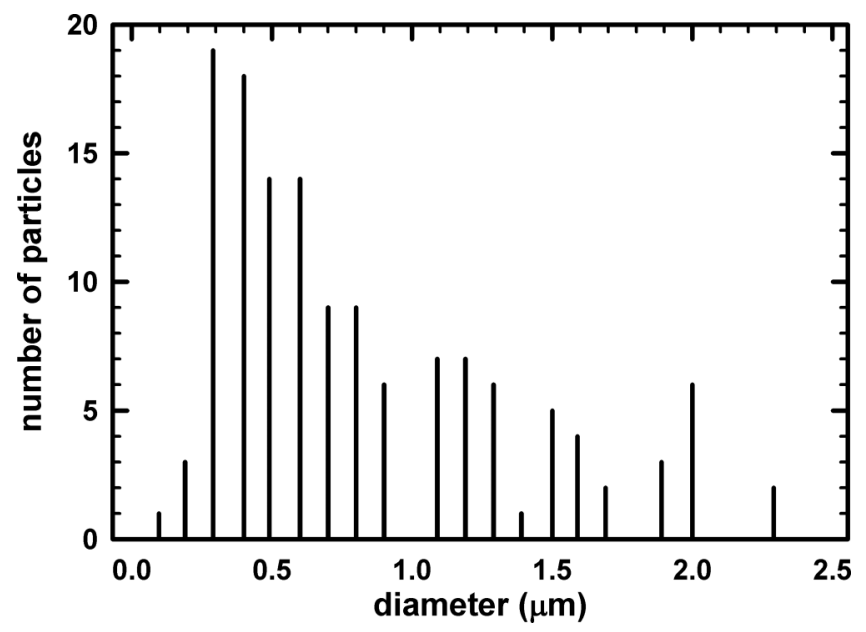

FIG. 1. Plot of the number of particles as a function of FGS diameter.

trajectory should reflect the tumbling motion, whereas for flexible crumpling objects a more constant angle but varying magnitude of the anisotropy should be obtained.

\section{EXPERIMENTAL METHODOLOGY}

\section{A. Materials}

Functionalized graphene sheets (FGSs) were mechanically dispersed in a $100-\mathrm{mP}$ as mineral oil (Sigma), which is Newtonian in the range of shear rates explored. Details of FGS synthesis was reported by McAllister et al. [49]. Functional epoxy and hydroxyl groups were present on the surfaces of the particles which effectively stabilize the sheets [50]. The viscosity of the suspending fluid was chosen such that the hydrodynamically dominated regime was accessible in the limited shear rate window of the rheometer. The average thickness and diameter of the FGSs are around 1.75 and $500 \mathrm{~nm}$, respectively. The results below indicate that the thickness of graphene particles is uniform, while large polydispersity was encountered in terms of equivalent diameters (by approximating the shape of the particles to circles) (see Fig. 1). Details on the atomic force microscopy characterization of the FGSs can be found elsewhere [21,49]. Five different concentrations of graphene sheets were prepared in the dilute regime [51]. The effective volume fractions (volume fraction of equivalent spheres with diameter equal to the average diameter of the FGSs) ranged between $2.3 \times 10^{-7}$ and $2.33 \times 10^{-6}$. The shear-rate-dependent dichroism normalized by number density results is found to be independent of volume fraction in the range explored, which confirms the dilute nature of the suspensions [21]. In the following sections the results presented are obtained with a particle volume fraction of $1.86 \times 10^{-6}$, which gave the optimal signal-to-noise ratio.

\section{B. Rheo-optics}

Rheo-optical techniques provide in situ information on the system microstructures in real time. The material property of interest is the refractive index tensor $\mathbf{n}=\mathbf{n}^{\prime}-i \mathbf{n}^{\prime \prime}$. Dichroism is related to differences in the principal eigenvalues of the imaginary part of the refractive index tensor $\Delta n^{\prime \prime}$. Physically, dichroism is related to the anisotropic attenuation in the transmitted light due to absorption and scattering. Dichroism measurements were performed using an in-house rheo-optical setup using MCR300 stress-controlled rheometer as the mechanical platform (Anton-Paar, Physica, Austria) [52]. A Couette cell was used with a laser light $(10-\mathrm{mW}$ He-Ne, $\lambda=632.8 \mathrm{~nm})$ being sent along the vorticity direction. This configuration allows the determination of flow-induced dichroism and 
orientation angle simultaneously as a function of applied shear rate. The inner and outer radii of the Couette cell are 16.95 and $17.95 \mathrm{~mm}\left(R_{i} / R_{o}=0.94\right)$, respectively, and the optical path length is $21 \mathrm{~mm}$. All experiments were performed at room temperature. The optical train consists of a laser $(\lambda=632.8 \mathrm{~nm})$, followed by a Glan-Thompson polarizer (Newport, USA), a photoelastic modulator (Beaglehole Instruments, New Zealand) operating at $50 \mathrm{kHz}$ and oriented at $45^{\circ}$ with respect to the polarizer and a zeroth-order quarter-wave plate (Newport) at $0^{\circ}$ with respect to the polarizer. Using a two-prism setup to guide the beam but designed so as to not change the polarization direction, the beam is passed into the Couette cell in the vorticity direction. The transmitted light is collected by a photodiode. Data from the photodiode is sent into two lock-in amplifiers (Stanford Research Systems Model 830) via a low-pass filter. The output from the lock-in amplifiers is the first and second harmonic signals $R_{1}$ and $R_{2}$. The setup is calibrated in such a manner that the orientation in the flow direction corresponds to a positive dichroism. The extinction coefficient $\delta^{\prime \prime}$ and the orientation angle $\chi$ are obtained from the first and second harmonics along with the calibration values for $J_{1}(A)$ and $J_{2}(A)$ (Bessel functions of the first kind) using Eqs. (1) and (3) [53]. The associated dichroism $\Delta n^{\prime \prime}$ is obtained from Eq. (2),

$$
\begin{gathered}
\delta^{\prime \prime}=-\operatorname{sgn}\left(R_{2}\right) \tanh ^{-1}\left\{\frac{1}{2} \sqrt{\frac{R_{1}}{-J_{1}(A)}+\frac{R_{2}}{-J_{2}(A)}}\right\}, \\
\Delta n^{\prime \prime}=\frac{\delta^{\prime \prime} \lambda}{2 \pi d},
\end{gathered}
$$

where $d$ is the optical path length and $\lambda$ the wavelength of the laser light source. The orientation angle is obtained from

$$
\chi=\frac{1}{2} \tan ^{-1}\left\{\frac{R_{1} / J_{1}(A)}{R_{2} / J_{2}(A)}\right\} .
$$

\section{KINETIC MODEL}

We consider a monodisperse suspension of rigid spheroidal particles dispersed in an incompressible Newtonian fluid. The suspension is sufficiently dilute such that the hydrodynamic interactions between particles can be neglected. The particles are force- and torque-free and they undergo Brownian motion. The shape of the particles can be defined in terms of aspect ratio $r$ or via the Bretherton constant [54] or form factor $\lambda$ defined by the ratio $\left(r^{2}-1\right) /\left(r^{2}+1\right)$. For very long thin rods $\lambda$ tends to 1 , while for thin spheroidal disks it is equal to -1 . The orientation of a single spheroid is described by a unit vector $\mathbf{p}$ parallel to its axis of revolution. For a thin disk $\mathbf{p}$ is directed perpendicularly to the surface of the disk. For a homogeneously imposed oscillatory shear flow, the time change of the single spheroid orientation vector follows the Jeffery equation [55]

$$
\dot{\mathbf{p}}=\boldsymbol{\Omega} \cdot \mathbf{p}+\lambda(\mathbf{E} \cdot \mathbf{p}-\mathbf{E}: \mathbf{p p p}),
$$

where $\mathbf{E}$ is the rate of deformation tensor and $\boldsymbol{\Omega}$ the vorticity tensor. The evolution described by (4) is deterministic. The randomness in the motion induced by Brownian motion requires a statistical description. One possibility is to employ the orientation distribution function $\psi(\mathbf{p}, t)$ defined as the probability to find a particle oriented within a solid angle $d \mathbf{p}$ of $\mathbf{p}$ is $\psi d \mathbf{p}$. The time evolution of $\psi$ follows the one of any convected scalar [56]

$$
\frac{\partial \psi}{\partial t}+\nabla \cdot\left(\dot{\mathbf{p}} \psi-D_{r} \nabla \psi\right)=0
$$

where $D_{r}$ is the rotational Brownian coefficient and $\nabla=\partial / \partial \mathbf{p}$ [48]. The term in parentheses represents the flux of the particle probability in the orientation space due to the torque induced by the external flow field and to the random effect of Brownian motion. A concise description of the orientation state of the system can be achieved by calculating the second-order orientation tensor 
defined as

$$
\mathbf{a}_{2}=\int \mathbf{p p} \psi d \mathbf{p}
$$

This tensor contains average information on the overall orientation state of the system. Following Fuller [53], we can express the average orientation angle with respect to the flow direction as a function of the orientation tensor

$$
\chi=\frac{1}{2} \arctan \left(\frac{2 a_{12}}{a_{11}-a_{22}}\right),
$$

where $a_{i j}$ are the components of $\mathbf{a}_{2}$. In $a_{i j}$, the subscripts 1, 2, and 3 indicates flow, gradient, and vorticity direction, respectively. Equation (5) is numerically solved since no simple analytical solution is possible. Asymptotic solutions are only available for spheroids in the limit of $r \approx 1[2,56]$. Here a finite-volume method is employed following Ferec and co-workers [57,58] (see Ref. [57] for more details about the mesh and the treatment of the periodic boundary conditions). A central scheme is implemented to interpolate properties between nodes. Once the orientation distribution is numerically solved, the orientation tensor is evaluated and the average orientation angle is obtained.

In the model, two input parameters are required: $\lambda$ and the rotational diffusion coefficient $D_{r}$. For the graphene suspension studied in the paper these two parameters have been obtained experimentally and $\lambda$ can be estimated from atomic force microscopy and $D_{r}$ from the dichroism relaxation measurements $[49,50]$. For the graphene particles in this work we approximate the sheets by very flat spheroidal objects with $\lambda=-1$. The polydispersity of the system had a negligible impact on $\lambda$ given its quadratic dependence on $r$. For the polydisperse graphene suspension the average rotational diffusion coefficient $D_{r}=0.04 \mathrm{~s}^{-1}$ was calculated from the dichroism relaxation measurements [51]. The rotational Brownian motion coefficient for thin disks in dilute regime scales as $D_{r} \backsim D^{-3}$, where $D$ is the diameter of the disk. This dependence and the large polydispersity in sizes observed in Fig. 1 warn us of the strong effect of polydispersity on the orientation dynamics of the system. The effect of polydispersity has been discussed in detail in another work [51]. Specifically, the dichroic response in simple steady shear was found to be dominated by the larger particles in the samples. This is a consequence of the fact that scattering intensity depends on the volume average over the ensemble. Here we settle for a simplified description by assuming a monodisperse sample with the averaged Brownian coefficient. It will be shown that this simplified description captures surprisingly well the system dynamics.

\section{RESULTS}

The experimental dichroism response to an oscillatory shear flow at $1 \mathrm{~Hz}$ corresponding to a Deborah number of about 26 is presented in Fig. 2. The flow around the particle is instantaneously developed, given that the timescale of the momentum diffusion $D^{2} / v$ is much smaller that the oscillation time $1 / \omega$. Here $v$ is the kinematic viscosity of the fluid.

The maximum Weissenberg number Wi was kept below 1.6 to compare with experiments, which give a ratio $\mathrm{Wi} / \mathrm{De} \approx 0.06$. At small Weissenberg number $(4 / 25)$ the average dichroism intensity of the loops is relatively small, as shown in Fig. 2. As the Weissenberg number is increased to $4 / 5$ and $8 / 5$, the intensity of the dichroism loops increases, showing the development of flow-induced orientation. A better way to visualize the same result is by plotting the average orientation angle with respect to the flow direction $\chi$, as shown in Fig. 3.

At $\mathrm{Wi}=4 / 25$ the orientation angle varies between $\pm 42^{\circ}$. A value of $45^{\circ}$ represents an isotropic orientation distribution in the system. Hence we are only slightly perturbing the orientation distribution of the suspension at $\mathrm{Wi}=4 / 25$. As we increase the imposed deformation, the amplitude of the $\chi$ loops decreases and the particle spends more time around the flow direction $\left(0^{\circ}\right)$. Interestingly, the particles do not fully tumble at high Wi. They oscillate around the flow direction, showing dynamics similar to the wagging dynamics found in nematic rod suspension in steady shear 


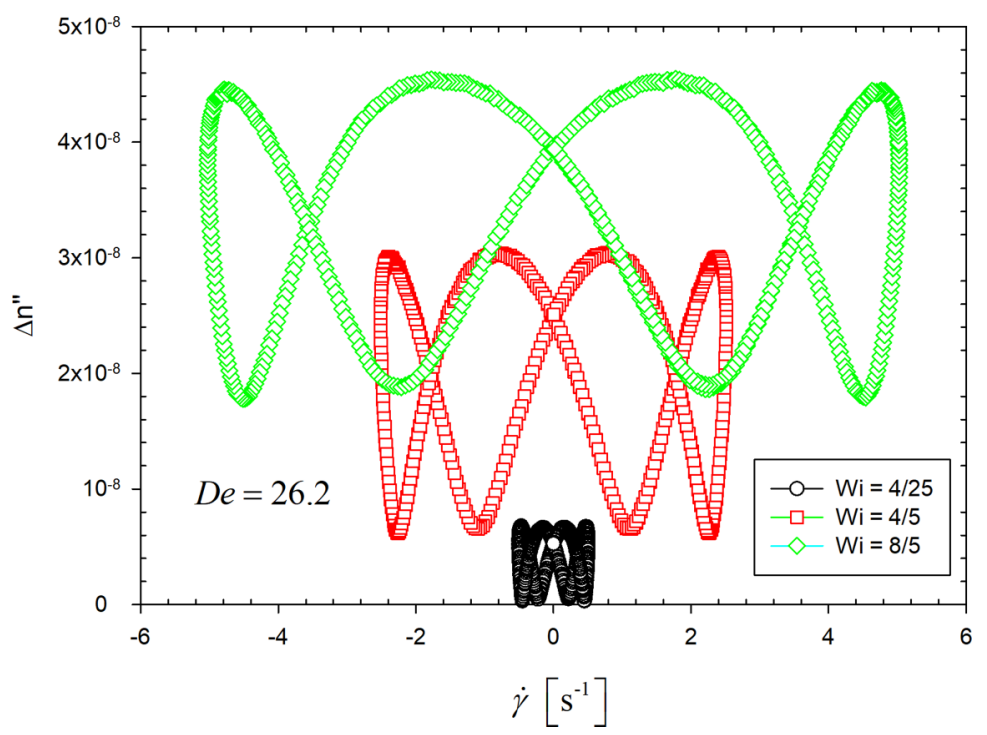

FIG. 2. Calculated loops from dichroism experiments versus shear rate for different Wi at $\mathrm{De}=26.2$ for a dilute suspension with $\lambda=-1$.

flow [59-61], which has been evidenced experimentally by flow dichroism and rheological measurements on polymeric rods [60,62]. In both Figs. 2 and 3 we report only the steady oscillatory response. The full evolution from the startup of the oscillatory flow is presented in Fig. 4, where the orientation angle is shown as a function of time.

In addition to the flow-induced alignment observed in Figs. 2 and 3, a double-peaked response can also be clearly seen in Fig. 4. This response is particularly interesting since its dynamics has double the frequency of the imposed oscillatory shear flow. In Fig. 4 the model predictions are also

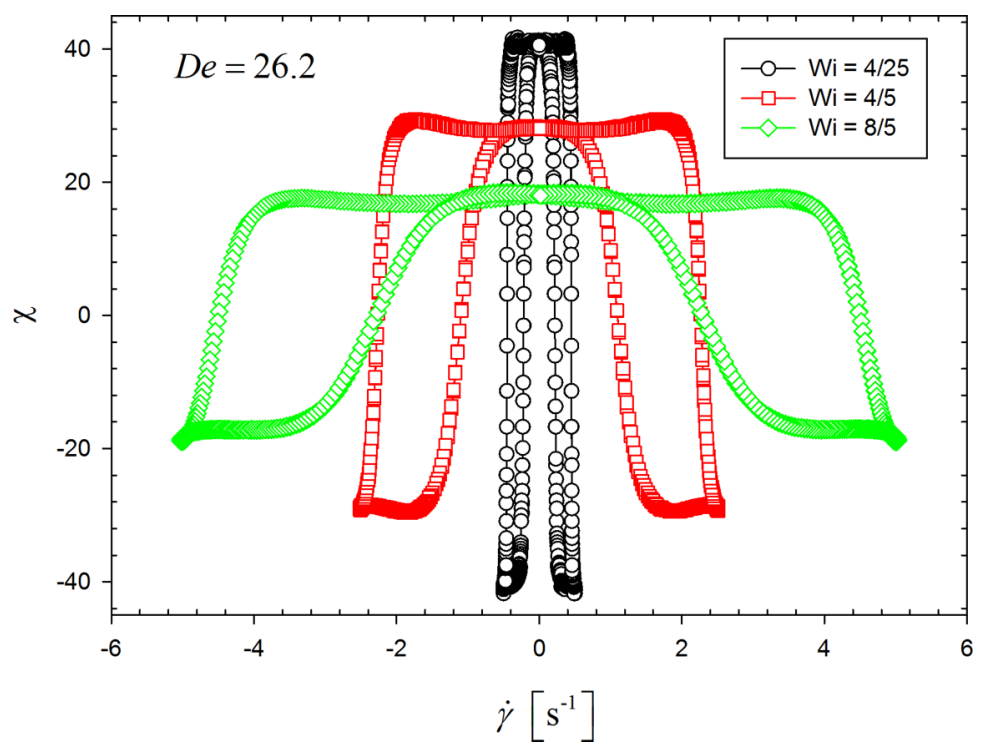

FIG. 3. Loops of the orientation angle versus shear rate for different $\mathrm{Wi}$ at $\mathrm{De}=26.2$ for a dilute suspension with $\lambda=-1$. 


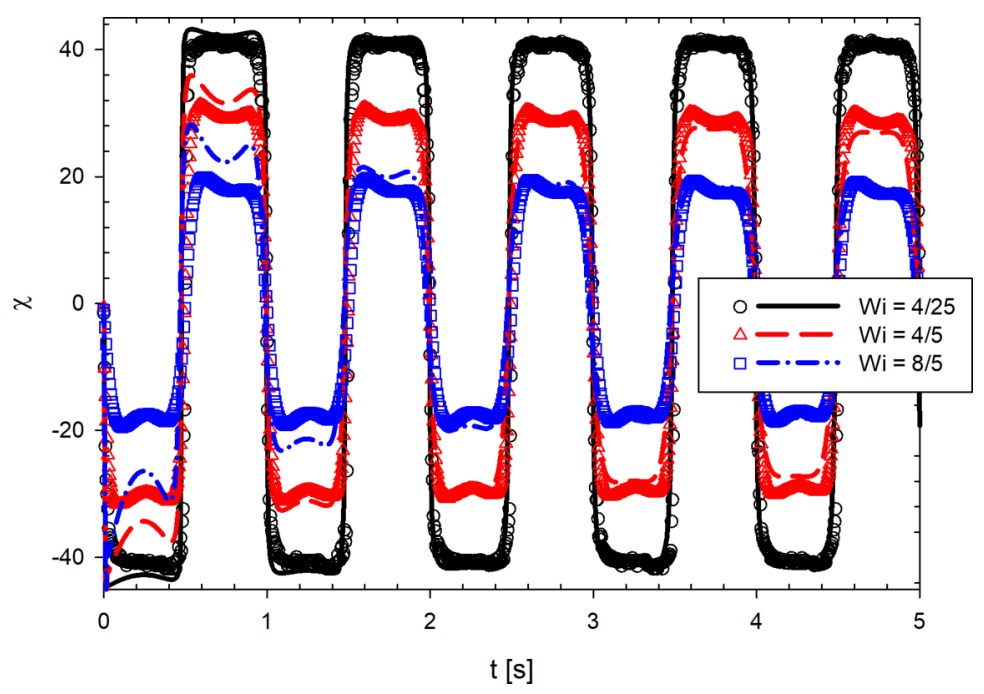

FIG. 4. Experimental orientation angle (symbols) and model predictions (lines) as a function of time for different Wi at De $=26.2$.

reported along with the experimental data. During the first period of oscillation, the model predicts a larger amplitude than in the experiments. The error increases for higher Wi. The difference seen in the first period of oscillation decays rapidly and already in the second period of oscillation, the model predictions are in excellent agreement with the experiments. The model presents a scenario where the particles in the orientation space get more and more synchronized as the oscillatory flow is applied. The large discrepancy in the first oscillatory cycle can be due to a series of factors. One of the main model assumptions is the monodispersity of the particles, whereas the system is experimentally polydisperse. Polydispersity can obfuscate the rotational dynamics of the individual particles because of phase mixing [19]. Since the period of the Jeffery orbit is a function of the particles aspect ratio, polydispersity will lead to a mixing of the transient dynamics of $\chi$.

To elucidate the microstructural cause behind this frequency doubling, it is useful to examine the components of the second-order orientation tensor obtained from the model. These components are directly connected to the average orientation angle according to Eq. (7). In Fig. 5 we report the components of $\mathbf{a}_{2}: a_{11}, a_{22}$, and $a_{12}$ for $\mathrm{Wi}=4 / 5$ and $\mathrm{De}=26.2$.

The evolution for the other Wi is qualitatively the same as the one shown in Fig. 5. Since the particles are treated as thin disks, the unit vector $\boldsymbol{p}$ is perpendicular to the disk surface. Hence for a perfectly aligned particle in the flow direction, the projection of $\boldsymbol{p}$ in the flow direction $p_{1}$ would be zero, while its projection in the gradient direction $p_{2}$ would be one. The components $a_{11}$ and $a_{22}$ provide an average quantification of the alignment of the particles. They are zero and one, respectively, for a perfectly aligned system and both equal to $1 / 3$ for an isotropic state. The component $a_{12}$ describes the average position of the particles in the flow-gradient plane. The change of sign of $a_{12}$ indicates that the particles are passing through the flow direction. The wagging behavior is confirmed here since the components $a_{11}$ and $a_{22}$ never go to zero (which would show collective tumbling dynamics) but they stay always positive. The steady-state oscillatory response is reached at around $4 \mathrm{~s}$, in agreement with the relation time $1 / 6 D_{r}$. Figure 5 also shows that the oscillations for the components $a_{11}$ and $a_{22}$ have frequency $2 \omega$, while $a_{12}$ oscillations have frequency $\omega$. Similar dependences were also obtained for slightly deformed spheroids in small-amplitude oscillatory shear [56] and in LAOS [2] via regular perturbation analysis. This double frequency for the normal components is also responsible for the double-peaked response of the orientation angle [see Eq. (7)] and it is a direct consequence of the wagging dynamics. During wagging, the particles oscillate between two symmetric configurations around the flow direction as shown in Fig. 6. For these two configurations the components of $a_{11}$ 


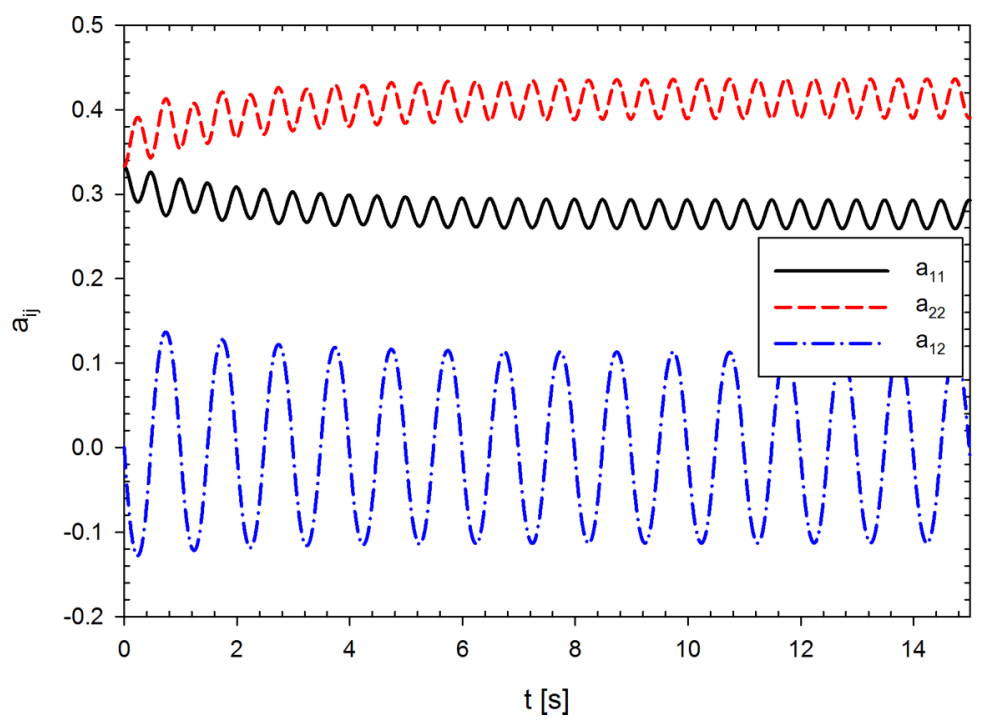

FIG. 5. Orientation tensor components as a function of time at $\mathrm{Wi}=4 / 5$ and $\mathrm{De}=26.2$.

and $a_{22}$ are equal, while $a_{12}$ is equal and opposite in sign, hence the double frequency for the normal components. The simple model that we have employed in this study thus captures dynamics in both small- and large-amplitude oscillatory flows.

In Fig. 7 the steady oscillatory dynamics of $a_{11}$ vs $a_{12}$ are given for Wi higher than the ones that could experimentally be probed while maintaining the same De. As the Wi increases, the loops move to lower values of $a_{11}$ since the particles are getting more and more aligned as the imposed deformation increases. The shape of the loops changes drastically for $\mathrm{Wi} \gg 1$. Analyzing the case $\mathrm{Wi}=16$, a clear recoil after flow reversal is observed for $a_{12}$. The dynamics is overall still a wagging dynamics but a secondary weak oscillation after flow reversal is observed. For this last case the ratio $\mathrm{Wi} / \mathrm{De}$ is equal to about 0.6 (lower than 1), implying that the system is still experiences low strains before flow reversal. In Fig. 8 the steady oscillatory dynamics of $a_{11}$ vs $a_{12}$ is reported for the same $\mathrm{Wi}$ and for $\mathrm{De}=1$. Also in this case we observe a critical change of the loop shapes for Wi $\gg 1$ and a weak recoil for $\mathrm{Wi} \geqslant 8$. The wider amplitude of oscillation for $a_{12}$ is a direct consequence of the larger deformations experienced by the system (the maximum ratio $\mathrm{Wi} / \mathrm{De}$ in this case is equal to 16), implying a larger wagging oscillation and an overall lower alignment in the flow direction (lower values of $a_{11}$ with respect to the case of $\mathrm{De}=26.2$ ).

One interesting observation we found from this study is the efficacy of the oscillatory flow to create alignment or misalignment as compared to steady shear flow [51], provided strain amplitudes are chosen carefully relative to the tumbling periods. In Figs. 9 and 10 the dichroism and orientation angle for the graphene suspension due to steady and oscillatory shear flow are reported. Here the Péclet

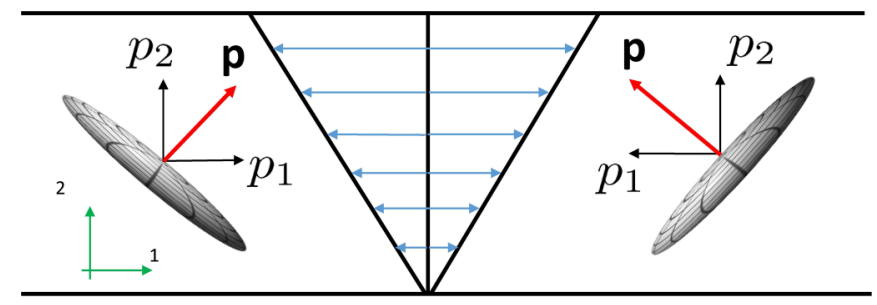

FIG. 6. Schematic of the two symmetric configurations assumed by the particles during oscillatory flow. 


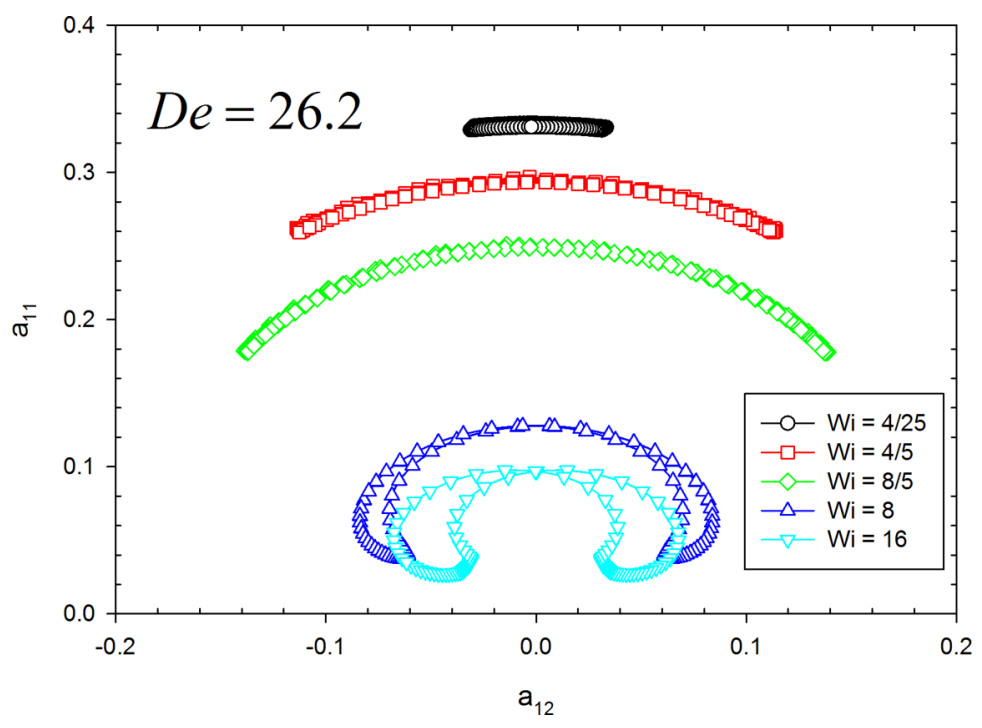

FIG. 7. Loops of the component $a_{11}$ of the orientation tensor as a function of the adimensional shear rate for different values of $\mathrm{Wi}$ at $\mathrm{De}=26.2$.

number Pe is defined as the ratio between the Brownian and hydrodynamic torques $\left(\mathrm{Pe}=\dot{\gamma} / D_{r}\right)$. For oscillatory flow, $\dot{\gamma}$ is equal to $\gamma_{0} \omega$ and the Péclet and Weissenberg numbers are not equal. Also, the values of dichroism and orientation angle are the maximum during the steady oscillations. In steady shear, dilute (and polydisperse) graphene suspensions show scalings similar to the one seen for single-walled and multiwalled carbon nanotubes $[22,63,64]$. We note here that the assumption of monodispersity of the model has a larger impact on the steady shear results. Indeed, the model would predict a more isotropic state at $\mathrm{Pe}<1$ than what is observed experimentally in Fig. 10. Higher degrees of orientation at low Pe are a consequence of the large polydispersity of our samples

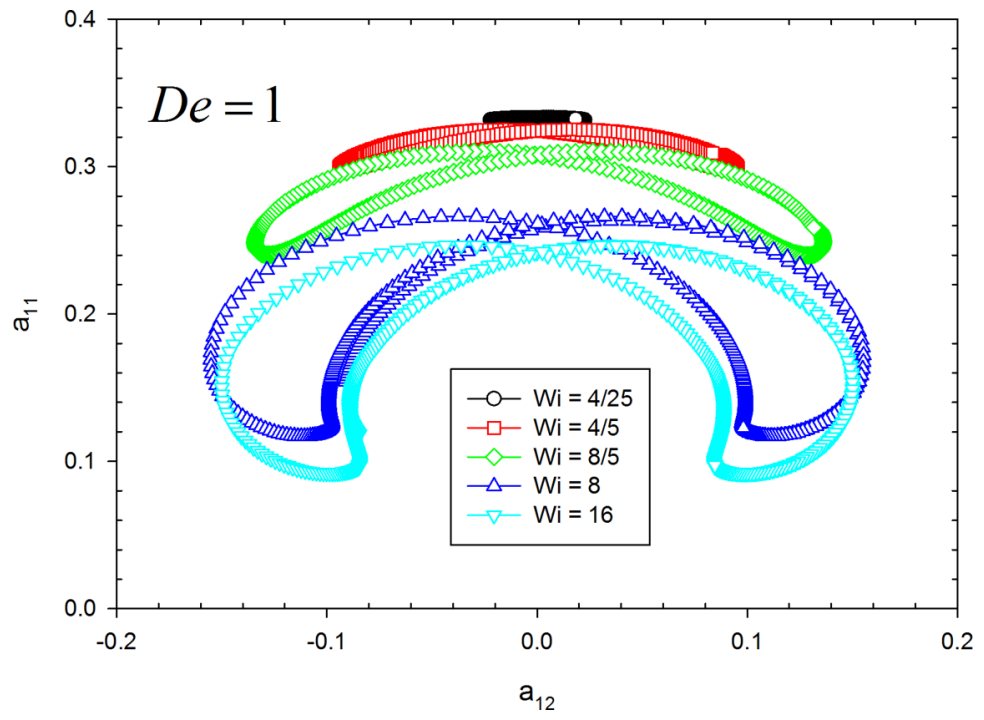

FIG. 8. Loops of the component $a_{11}$ of the orientation tensor as a function of the adimensional shear rate for different values of Wi at $\mathrm{De}=1$. 


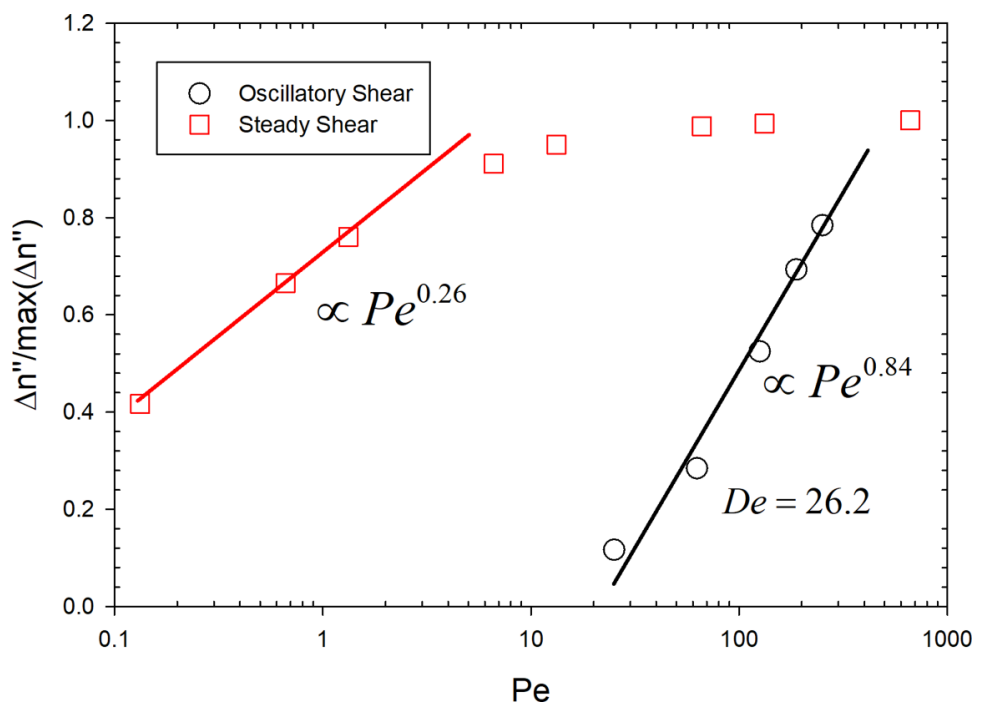

FIG. 9. Comparison between dichroism values obtained in steady-state shear (square) and oscillatory shear (circle) versus Pe. For oscillatory shear the maximum value during the oscillation was used as an indicator.

together with the contribution of larger particles to the scattering intensity and therefore to the overall dichroic signal.

In steady oscillatory flows, the scaling departs from the one observed in steady shear. At equivalent $\mathrm{Pe}$, a lower level of anisotropy in oscillatory flow is observed in comparison with steady shear flow. However, the microstructures are more sensitive to change in oscillatory flow than in shear flow. Recently, Khair [2] developed an asymptotic solution for slightly deformed spheroids $(\lambda \simeq 0)$ in

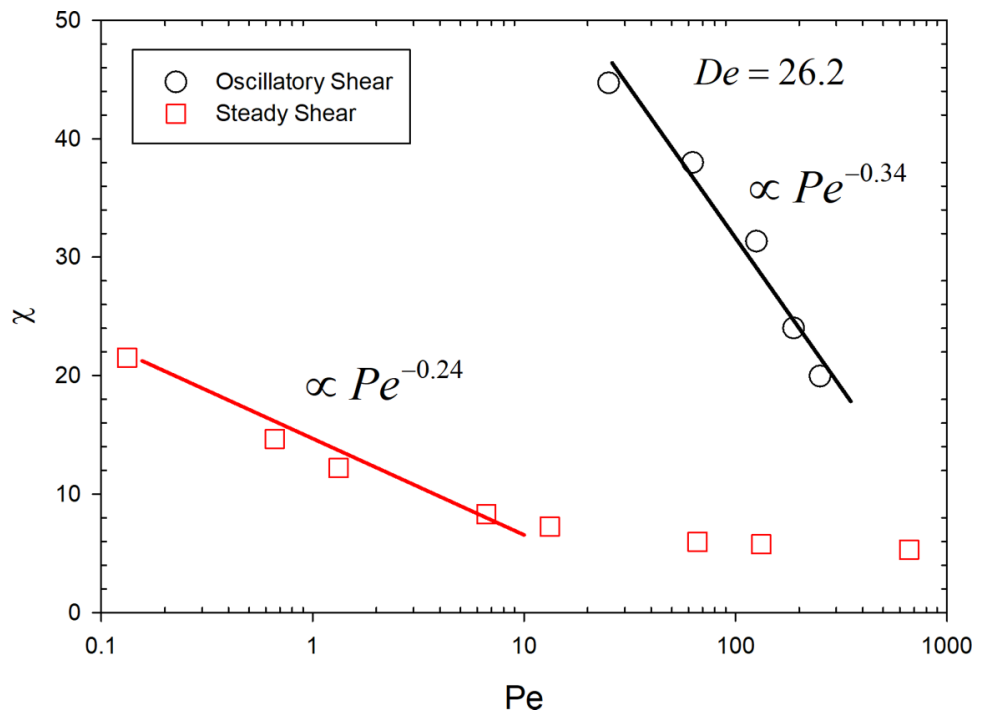

FIG. 10. Comparison between orientation angles obtained in steady-state shear (square) and oscillatory shear (circle) as a function of Pe. For oscillatory shear the maximum value during the oscillation was used as an indicator. 
LAOS that predicted different scalings for $\chi$ between steady and oscillatory shear flow, which is in qualitative agreement with our findings.

\section{CONCLUSION}

We have experimentally studied the orientation dynamics of functionalized graphene suspensions in oscillatory shear flow. The experimental observations were compared to a model with a singleparticle Smoluchowski equation, allowing us to obtain insights into the nonlinear dynamics of a complex fluid in time-dependent oscillatory shear flow. In agreement with expectation from scaling theory for 2D sheets, the molecularly thin functionalized graphene sheets act as rigid flat particles. Interestingly, a frequency doubling was observed. Microstructurally, this is caused by the normal components of the orientation tensor $\left(a_{11}\right.$ and $\left.a_{22}\right)$ that oscillates with frequency $2 \omega$ while the cross component $a_{12}$ oscillates with frequency $\omega$. This double frequency of the normal components of the orientation tensor also implies that the normal stresses would oscillate with double the frequency of the applied shear stress [2,56]. Moreover, the phase mixing effect observed in Fig. 4 may provide a simple alternative to determine polydispersity of a suspension directly from dichroism measurements, following earlier work on phase mixing in steady-state flows [19]. At large Wi, the particles were found to follow a wagging dynamics and in LAOS, a secondary recoil was also observed in the model predictions. Finally, the orientation dynamics of the microstructures were found to be more sensitive to the oscillatory flow as compared to the steady shear flow. Thus oscillatory flow presents a possibility to differentiate particles dynamics at high Pe, which is not possible by applying steady shear flow.

\section{ACKNOWLEDGMENTS}

J.V. acknowledges the Swiss National Science Foundation Project No. 200021-157147 and G.N. acknowledges funding from the NSERC Grant No. RGPIN-2017-03783.

[1] A. S. Khair, Large amplitude oscillatory shear of the Giesekus model, J. Rheol. 60, 257 (2016).

[2] A. S. Khair, On a suspension of nearly spherical colloidal particles under large-amplitude oscillatory shear flow, J. Fluid Mech. 791, R5 (2016).

[3] K. Hyun, M. Wilhelm, C. O. Klein, K. S. Cho, J. G. Nam, K. H. Ahn, S. J. Lee, R. E. Ewoldt, and G. H. McKinley, A review of nonlinear oscillatory shear tests: Analysis and application of large amplitude oscillatory shear (LAOS), Prog. Polym. Sci. 36, 1697 (2011).

[4] A. K. Geim, Graphene: Status and prospects, Science 324, 1530 (2009).

[5] A. D. Schlüter, P. Payamyar, and H. C. Öttinger, How the world changes by going from one to two dimensional polymers in solution, Macromol. Rapid Commun. 37, 1638 (2016).

[6] C. Lee, X. Wei, J. W. Kysar, and J. Hone, Strength of monolayer graphene measurement of the elastic properties and intrinsic, Science 321, 385 (2008).

[7] A. A. Balandin, S. Ghosh, W. Bao, I. Calizo, D. Teweldebrhan, F. Miao, and C. N. Lau, Superior thermal conductivity of single-layer graphene, Nano Lett. 8, 902 (2008).

[8] M. J. Allen, V. C. Tung, and R. B. Kaner, Honeycomb carbon: A review of graphene, Chem. Rev. 110, 132 (2010).

[9] H. Kim, A. A. Abdala, and C. W. Macosko, Graphene/polymer nanocomposites, Macromolecules 43, 6515 (2010).

[10] J. Shim, J. M. Yun, T. Yun, P. Kim, K. E. Lee, W. J. Lee, R. Ryoo, D. J. Pine, G.-R. Yi, and S. O. Kim, Two-minute assembly of pristine large-area graphene based films, Nano Lett. 14, 1388 (2014). 
[11] C. M. Weber, D. M. Eisele, J. P. Rabe, Y. Liang, X. Feng, L. Zhi, K. Mullen, J. L. Lyon, R. Williams, D. A. Vanden Bout, and K. J. Stevenson, Graphene-based optically transparent electrodes for spectroelectrochemistry in the UV-Vis region, Small 6, 184 (2010).

[12] L. Imperiali, K.-H. Liao, C. Clasen, J. Fransaer, C. W. Macosko, and J. Vermant, Interfacial rheology and structure of tiled graphene oxide sheets, Langmuir 28, 7990 (2012).

[13] X. Kang, J. Wang, H. Wu, J. Liu, I. A. Aksay, and Y. Lin, A graphene-based electrochemical sensor for sensitive detection of paracetamol, Talanta 81, 754 (2010).

[14] S. Stankovich, D. A. Dikin, G. H. B. Dommett, K. M. Kohlhaas, E. J. Zimney, E. A. Stach, R. D. Piner, S. T. Nguyen, and R. S. Ruoff, Graphene-based composite materials, Nature (London) 442, 282 (2006).

[15] P. L. Frattini and G. G. Fuller, The dynamics of dilute colloidal suspensions subject to time-dependent flow-fields by conservative dichroism, J. Colloid Interface Sci. 2, 506 (1984).

[16] P. L. Frattini and G. G. Fuller, Conservative dichroism of a sheared suspension in the Rayleigh-Gans light-scattering approximation, J. Colloid Interface Sci. 119, 335 (1987).

[17] A. Okagawa and S. G. Mason, The kinetics of flowing dispersions. VII. Oscillatory behavior of rods and discs in shear flow, J. Colloid Interface Sci. 45, 330 (1973).

[18] S. J. Johnson, P. L. Frattini, and G. G. Fuller, Simultaneous dichroism and birefringence measurements of dilute colloidal suspensions in transient shear flow, J. Colloid Interface Sci. 104, 440 (1985).

[19] J. Vermant, H. Yang, and G. G. Fuller, Rheooptical determination of aspect ratio and polydispersity of nonspherical particles, AIChE J. 47, 790 (2001).

[20] D. Z. Gunes, R. Scirocco, J. Mewis, and J. Vermant, Flow-induced orientation of non-spherical particles: Effect of aspect ratio and medium rheology, J. Non-Newtonian Fluid Mech. 155, 39 (2008).

[21] N. K. Reddy, J. Pérez-Juste, I. Pastoriza-Santos, P. R. Lang, J. K. G. Dhont, L. M. Liz-Marzán, and J. Vermant, Flow dichroism as a reliable method to measure the hydrodynamic aspect ratio of gold nanoparticles, ACS Nano 5, 4935 (2011).

[22] G. Natale, N. K. Reddy, G. Ausias, J. Férec, M. C. Heuzey, and P. Carreau, Rheo-optical response of carbon nanotube suspensions, J. Rheol. 59, 499 (2015).

[23] U. Reinhardt, E. L. Meyer, G. G. Fuller, and W. Kulicke, Rheo-optical characterization (flow-birefringence and flow-dichroism) of the tobacco mosaic virus, Macromol. Chem. Phys. 196, 63 (1995).

[24] T. A. J. Lenstra and J. K. G. Dhont, Flow dichroism in critical colloidal fluids, Phys. Rev. E 63, 061401 (2001).

[25] C. Mobuchon, M. C. Heuzey, P. J. Carreau, N. K. Reddy, and J. Vermant, Anisotropy of non-aqueous layered silicate suspensions subjected to shear flow, J. Rheol. 53, 517 (2009).

[26] L. Hilliou, D. Vlassopoulos, and M. Rehahn, Dynamics of hairy-rod polymer solutions in simple shear flow: Aging effects, Macromolecules 33, 3105 (2000).

[27] L. Hilliou, D. Vlassopoulos, and M. Rehahn, Dynamics of nondilute hairy-rod polymer solutions in simple shear flow, Macromolecules 34, 1742 (2001).

[28] B. D. Bedford and W. R. Burghardt, Molecular orientation and instability in plane Poiseuille flow of a liquid-crystalline polymer, J. Rheol. 38, 1657 (1994).

[29] J. M. Li and W. R. Burghardt, Flow birefringence in axisymmetric geometries, J. Rheol. 39, 743 (1995).

[30] S. A. Rogers, B. M. Erwin, D. Vlassopoulos, and M. Cloitre, A sequence of physical processes determined and quantified in LAOS: Application to a yield stress fluid, J. Rheol. 55, 435 (2011).

[31] K. Atalik and R. Keunings, On the occurrence of even harmonics in the shear stress response of viscoelastic fluids in large amplitude oscillatory shear, J. Non-Newtonian Fluid Mech. 122, 107 (2004).

[32] R. H. Ewoldt, A. E. Hosoi, and G. H. McKinley, New measures for characterizing nonlinear viscoelasticity in large amplitude oscillatory shear, J. Rheol. 52, 1427 (2008).

[33] S. A. Rogers and M. P. Lettinga, A sequence of physical processes determined and quantified in largeamplitude oscillatory shear (LAOS): Application to theoretical nonlinear models, J. Rheol. 56, 1 (2012).

[34] J. E. Bae and K. S. Cho, Semianalytical methods for the determination of the nonlinear parameter of nonlinear viscoelastic constitutive equations from LAOS data, J. Rheol. 59, 525 (2015).

[35] J. G. Nam, K. Hyun, K. H. Ahn, and S. J. Lee, Prediction of normal stresses under large amplitude oscillatory shear flow, J. Non-Newtonian Fluid Mech. 150, 1 (2008). 
[36] J. G. Nam, K. H. Ahn, S. J. Lee, and K. Hyun, First normal stress difference of entangled polymer solutions in large amplitude oscillatory shear flow, J. Rheol. 54, 1243 (2010).

[37] A. Calin, M. Wilhelm, and C. Balan, Determination of the non-linear parameter (mobility factor) of the Giesekus constitutive model using LAOS procedure, J. Non-Newtonian Fluid Mech. 165, 1564 (2010).

[38] R. H. Ewoldt and G. H. McKinley, On secondary loops in LAOS via self-intersection of Lissajous-Bowditch curves, Rheol. Acta 49, 213 (2010).

[39] A. Kate Gurnon and N. J. Wagner, Large amplitude oscillatory shear (LAOS) measurements to obtain constitutive equation model parameters: Giesekus model of banding and nonbanding wormlike micelles, J. Rheol. 56, 333 (2012).

[40] A. J. Giacomin, R. B. Bird, L. M. Johnson, and A. W. Mix, Large-amplitude oscillatory shear flow from the corotational Maxwell model, J. Non-Newtonian Fluid Mech. 166, 1081 (2011).

[41] D. S. Pearson and W. E. Rochefort, Behavior of concentrated polystyrene solutions in large-amplitude oscillating shear fields, J. Polym. Sci. B 20, 83 (1982).

[42] P. Vlahovska, J. Blawzdziewicz, and M. Loewenberg, Nonlinear rheology of a dilute emulsion of surfactantcovered spherical drops in time-dependent flows, J. Fluid Mech. 463, 1 (2002).

[43] Y. Renardy, Numerical simulation of a drop undergoing large amplitude oscillatory shear, Rheol. Acta 45, 223 (2006).

[44] R. B. Bird, A. J. Giacomin, A. M. Schmalzer, and C. Aumnate, Dilute rigid dumbbell suspensions in large-amplitude oscillatory shear flow: Shear stress response, J. Chem. Phys. 140, 074904 (2014).

[45] J. K. Dhont and W. J. Briels, Viscoelasticity of suspensions of long, rigid rods, Colloids Surf. A 213, 131 (2003).

[46] Y. Bozorgi and P. T. Underhill, Large-amplitude oscillatory shear rheology of dilute active suspensions, Rheol. Acta 53, 899 (2014).

[47] B. D. Leahy, D. L. Koch, and I. Cohen, The effect of shear flow on the rotational diffusion of a single axisymmetric particle, J. Fluid Mech. 772, 42 (2015).

[48] R. B. Bird, C. F. Curtiss, R. C. Armstrong, and O. Hassager, Dynamics of Polymeric Liquids, Vol. 2 Kinetic Theory, 2nd ed. (John Wiley \& Sons, New York, 1987).

[49] M. J. McAllister, J.-L. Li, D. H. Adamson, H. C. Schniepp, A. A. Abdala, J. Liu, M. Herrera-Alonso, D. L. Milius, R. Car, R. K. Prud'homme, and I. A. Aksay, Single sheet functionalized graphene by oxidation and thermal expansion of graphite, Chem. Mater. 19, 4396 (2007).

[50] H. C. Schniepp, J. Li, M. J. McAllister, H. Sai, M. Herrera-Alonso, D. H. Adamson, R. K. Prud'homme, R. Car, D. A. Saville, and I. A. Aksay, Functionalized single graphene sheets derived from splitting graphite oxide, J. Phys. Chem. B 110, 8535 (2006).

[51] N. K. Reddy, G. Natale, R. K. Prud'homme, and J. Vermant, Rheo-optical analysis of functionalised graphene suspensions, Langmuir (2018), doi: 10.1021/acs.langmuir.8b01574.

[52] L. Pellens, J. Vermant, and J. Mewis, Deviations from the stress-optical relation in telechelic associative polymer solutions, Macromolecules 38, 1911 (2005).

[53] G. G. Fuller, Optical Rheometry of Complex Fluids (Oxford University Press, Oxford, 1995).

[54] F. P. Bretherton, The motion of rigid particles in a shear flow at low Reynolds number, J. Fluid Mech. 14, 284 (1962).

[55] G. B. Jeffery, The motion of ellipsoidal particles immersed in a viscous fluid, Proc. R. Soc. London Ser. A 102, 161 (1922).

[56] L. G. Leal and E. J. Hinch, The rheology of a suspension of nearly spherical particles subject to Brownian rotations, J. Fluid Mech. 55, 745 (1972).

[57] J. Ferec, M. Heniche, M. C. Heuzey, G. Ausias, and P. J. Carreau, Numerical solution of the Fokker-Planck equation for fiber suspensions: Application to the Folgar-Tucker-Lipscomb model, J. Non-Newtonian Fluid Mech. 155, 20 (2008).

[58] G. Natale, M. C. Heuzey, P. J. Carreau, G. Ausias, and J. Ferec, Rheological modeling of carbon nanotube suspensions with rod-rod interactions, AIChE J. 60, 1476 (2014).

[59] R. G. Larson and H. C. Ottinger, Effect of molecular elasticity on out-of-plane orientations in shearing flows of liquid-crystalline polymers, Macromolecules 24, 6270 (1991). 
[60] J. Mewis, M. Mortier, J. Vermant, and P. Moldenaers, Experimental evidence for the existence of a wagging regime in polymeric liquid crystals, Macromolecules 30, 1323 (1997).

[61] V. Faraoni, M. Grosso, S. Crescitelli, and P. L. Maffettone, The rigid-rod model for nematic polymers: An analysis of the shear flow problem, J. Rheol. 43, 829 (1999).

[62] M. Grosso, S. Crescitelli, E. Somma, J. Vermant, P. Moldenaers, and P. L. Maffettone, Prediction and Observation of Sustained Oscillations in a Sheared Liquid Crystalline Polymer, Phys. Rev. Lett. 90, 098304 (2003).

[63] D. Fry, B. Langhorst, H. Kim, E. Grulke, H. Wang, and E. K. Hobbie, Anisotropy of Sheared CarbonNanotube Suspensions, Phys. Rev. Lett. 95, 038304 (2005).

[64] D. Fry, B. Langhorst, H. Wang, M. L. Becker, B. J. Bauer, E. Grulke, and E. K. Hobbie, Rheo-optical studies of carbon nanotube suspensions, J. Chem. Phys. 124, 054703 (2006). 